Article

\title{
Controlling the Skin Barrier Quality through the Application of Polymeric Films Containing Microspheres with Encapsulated Plant Extract
}

\author{
Justyna Kozlowska ${ }^{1, *(D)}$, Bartosz Tylkowski ${ }^{2}$ (D), Natalia Stachowiak ${ }^{1}$ and \\ Weronika Prus-Walendziak ${ }^{1}$ \\ 1 Faculty of Chemistry, Nicolaus Copernicus University in Torun, Gagarina 7, 87-100 Torun, Poland; \\ nat.sta@doktorant.umk.pl (N.S.); weronika.prus.walendziak@gmail.com (W.P.-W.) \\ 2 Eurecat, Centre Tecnològic de Catalunya, C/Marcellí Domingo s/n, 43007 Tarragona, Spain; \\ bartosz.tylkowski@eurecat.org \\ * Correspondence: justynak@chem.umk.pl
}

Received: 19 March 2020; Accepted: 28 April 2020; Published: 30 April 2020

check for updates

\begin{abstract}
Human skin has protective functions and it is a barrier that protects the interior of the body from harmful environmental factors and pathogen penetration. An important role of the skin is also to prevent the loss of water from the body and if the skin barrier is damaged, the amount of water emitted from the internal environment is increased. Therefore, it is crucial to recovery and maintenance of epidermal barrier integrity. The aim of the current work was to encapsulate Calendula officinalis flower extract in gelatin microspheres and then incorporation microspheres into thin polymeric films made from sodium alginate or mixture of sodium alginate and starch. Such materials may find applications in the cosmetic field for example in the preparation of masks for skin, according to the Calendula officinalis flower extract wide influence on skin condition. Thus, the release profile of this extract from the materials was tested under conditions corresponding to the skin ( $\mathrm{pH} 5.4,37^{\circ} \mathrm{C}$ ). The mechanical properties, surface free energy, and moisture content of obtained films were measured. To determine the barrier quality of the stratum corneum, transepidermal water loss (TEWL) and skin color measurements were performed. The loaded microspheres were successfully incorporated into polymeric films without affecting its useful properties. Although the values of Young's modulus and the moisture content were decreased after film modification by microspheres addition, the skin parameters were much better after application of films with microspheres. The results confirmed that obtained materials can be potentially used in cosmetics to improve the skin barrier quality.
\end{abstract}

Keywords: polymer films; microspheres; Calendula officinalis flower extract; encapsulation; sodium alginate; starch; skin barrier; transepidermal water loss

\section{Introduction}

The skin is the body's largest organ and it is a barrier between the internal physiological environment and the external environment. The barrier function of the skin is very important for our health to prevent invasion of pathogenic microorganisms. Moreover, the human skin has a major function to limit water evaporation from the body. The barrier properties are assured by the stratum corneum, which controls the transepidermal water loss (TEWL). The level of TEWL is used as an indicator of permeability of the skin and therefore it indicates skin barrier function. Low-humidity weather conditions, skin injuries and abrasions, as well as topically applied products that dry out the skin, such as soap and detergents, can affect TEWL. As it is known, in the case of weakened barrier function higher values of TEWL are observed, whereas decreased TEWL proves an unimpaired 
skin barrier or its repair. Dry skin shows increased TEWL and the use of moisturizing products is recommended to improve skin hydration [1-4].

Natural hydrophilic polysaccharides and proteins such as sodium alginate, starch, and gelatin are widely used in cosmetics. These polymers are considered to be a promising and beneficial substances in the production of useful materials due to their thickening, gelling and film-forming properties, relatively low price, and abundance [5-7].

Sodium alginate is a linear copolymer consisting blocks of (1-4)-linked $\beta$-D-mannuronate and $\alpha$-L-guluronate residues. This anionic polysaccharide is distributed in the cell walls of brown algae (Phaeophyceae) [7,8]. Another popular polysaccharide is starch obtained mainly from potato tubers. Starch is used in most green plants as energy storage. Its structure is composed of two glucans-linear amylose and branched amylopectin, and is stabilized by hydrogen bonds between hydroxyl groups [9-11]. Gelatin is a denatured, biodegradable protein obtained by processing of collagen extracted from animal tissue such as skin, muscle, and bone. Gelatin used commercially is predominantly obtained from bovine or porcine skin and bones, which are subjected to partial acid hydrolysis (giving gelatin type A) or partial alkaline hydrolysis (leading to gelatin type B) [12,13].

Biopolymers are also used in many encapsulation techniques. Microencapsulation technology is crucial in the field of food industry in order to protect and stabilize oxidizable substrates such as fats and oils. This technology was recognized a long time ago in medicine and pharmaceutical industry for drug delivery. Its cosmetic and agriculture applications are also more common. Microencapsulation is employed to enclose in polymeric or inorganic structures various types of solid and liquid materials such as drugs, extracts, vitamins, perfumes, proteins, dyes, bacterial cells, etc. [14-16].

Calendula officinalis $\mathrm{L}$. is a medical plant and the main constituents of its flower extract include carotenoids, terpenoids, steroids, phenolic acids, and flavonoids like quercetin, rutin, narcissi, isorhamnetin, and kaempferol [17]. Calendula officinalis flower extract (pot marigold flower extract) has been determined to exhibit beneficial effects on humans such as antioxidant $[18,19]$, anti-inflammatory [20], antimicrobial [21], anti-cancer, and anti-viral activity [22]. For that reason, it is used in treatment of burns, ulcers, skin inflammations, eczema, bruises, cuts, rashes, skin wounds, and other conditions $[19,23,24]$. The beneficial effects of Calendula officinalis extract on human health can be enhanced by controlling its release from polymeric microspheres.

The aim of our study is to prepare materials in the form of biopolymer films with the addition of microspheres containing plant extract. We would like to obtain new advanced composite materials for cosmetic applications to improve the barrier function of stratum corneum by combining microspheres, which are the carriers of the active ingredients, and the polymeric matrix made from hydrophilic polymers with the addition of glycerol. The biopolymers used in this work were gelatin-to obtain microspheres, as well as sodium alginate and starch - to obtain the polymer matrices. We have prepared the gelatin microspheres tested in our laboratory previously, which were at first incorporated into porous three-dimensional matrices made from collagen, gelatin, and hydroxyethyl cellulose [25-27]. This time, we decided to investigate how these types of microspheres will work after incorporation into polymer thin films. We expected an improvement in the penetration of the active substance into the skin. For this purpose, besides mechanical properties, moisture content, contact angles, and in vitro release study, the preliminary tests with the usage of a tewameter and a colorimeter were carried out. Such films could be used on the skin, for example as cosmetic masks.

\section{Materials and Methods}

\subsection{Materials}

Starch from potato (S), gelatin type A from porcine skin, 1-ethyl-3(3-dimethylaminopropyl) carbodiimide (EDC), N-hydroxysuccinimide (NHS), Span 85, Folin-Ciocalteu reagent, and gallic acid were purchased from Sigma-Aldrich (Poznan, Poland). Sodium alginate (ALG) was supplied from BÜCHI Labortechnik AG (Flawil, Switzerland). Glycerol (G) was supplied by the Stanlab 
(Lublin, Poland). All the other reagents were obtained from Avantor Performance Materials Poland S.A. (Gliwice, Poland). All used chemicals were of analytical grade. The hydroglycolic Calendula officinalis flower extract (propylene glycol/water (80:20)) was acquired from Provital S.A. (Barcelona, Spain). The total polyphenol content of Calendula officinalis flower extract was determined using the Folin-Ciocalteu method [28] with gallic acid as a standard. The absorbance measurements were performed at $725 \mathrm{~nm}$ using a UV-VIS spectrophotometer. The results were calculated as gallic acid equivalents (mgGAE/mL). The total polyphenolic content of the extract was $206.6 \pm 3.6 \mathrm{mgGAE} / \mathrm{mL}$.

\subsection{Preparation of Gelatin Microspheres}

The gelatin microspheres were obtained using the method detailed in our previous paper with some modifications [25]. Briefly, microspheres were produced from $20 \%(w / w)$ aqueous gelatin solution. Firstly, gelatin solution was heated at $40^{\circ} \mathrm{C}$ for $30 \mathrm{~min}$ and then $40 \mathrm{~mL}$ of paraffin oil with the addition of $1 \%$ Span 85 was appended. The mixture was homogenized for $20 \mathrm{~min}$ (T25 digital ULTRA-TURRAX disperser, IKA Werke, Staufen, Germany) and the obtained water-in-oil emulsion was magnetically stirred for $2 \mathrm{~h}$. To improve physical properties of the gelatin microspheres, EDC/NHS mixture was used as a crosslinking agent. After centrifugation and washing with ethanol, the microspheres were frozen and lyophilized (ALPHA 1-2 LD plus lyophilizator, Martin Christ, Osterode am Harz, Germany).

The Calendula officinalis flower extract was loaded into the microspheres by immersion in $2 \% v / v$ commercial hydroglycolic extract for $24 \mathrm{~h}$ and incubation at $37^{\circ} \mathrm{C}$ for $24 \mathrm{~h}$. Then, any remaining extract was removed, and the microspheres were washed with deionized water, frozen, and lyophilized. The scheme of microspheres production by a water-in-oil emulsion method is shown in Figure 1.

gelatin type A solution

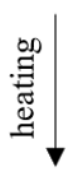

aquoeus polymer phase

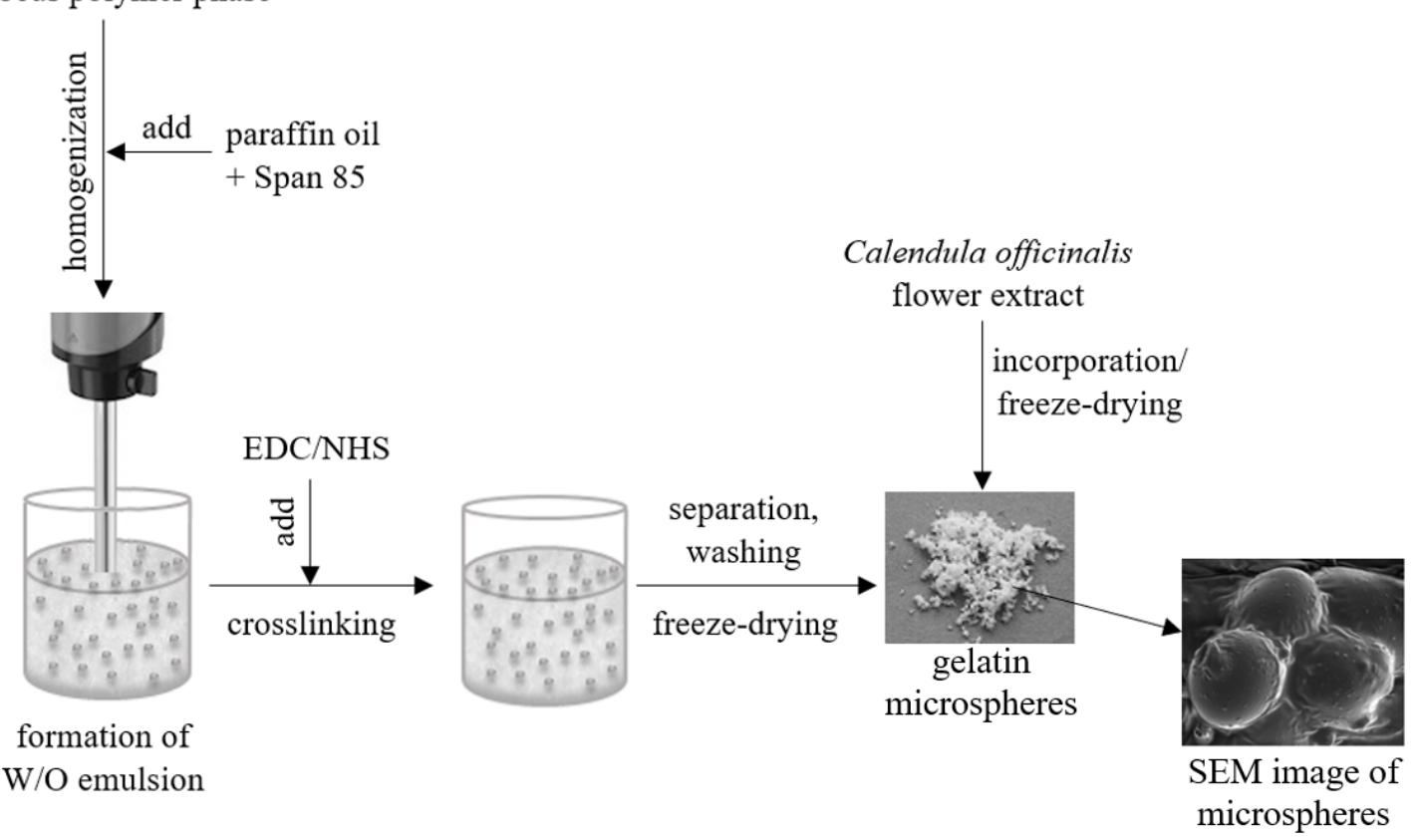

Figure 1. The scheme of production of gelatin microspheres loaded with Calendula officinalis flower extract. 


\subsection{Production of Polymeric Films}

The polymer films were produced from sodium alginate and glycerol or sodium alginate, starch, and glycerol (Table 1). Firstly, sodium alginate and starch were dissolved in deionized water, to prepare $2 \%(w / w)$ of sodium alginate solution and $0.5 \%(w / w)$ of starch solution. After that, polymer blends were prepared by mixing these solutions in different weight ratios and various amounts of glycerol were added to the mixtures (Figure 2). The films were also fabricated from sodium alginate solution without the addition of starch. Thin films were obtained by solution casting on glass plates and the samples were dried at room temperature. Moreover, this method was repeated for obtaining samples containing $0.6 \%$ of gelatin microspheres loaded with pot marigold flower extract. The prepared mixtures were homogenized before casting.

Table 1. The composition of the prepared films (ALG—sodium alginate, S—starch, G—glycerol).

\begin{tabular}{ccccc}
\hline & & \multicolumn{2}{c}{ Weight Ratio (\%) } & Addition (\%) \\
\hline & Sample & ALG & S & G \\
\hline 1. & ALG + 1.5 G & 100 & - & 1.5 \\
2. & ALG + 2.0 G & 100 & - & 2.0 \\
3. & ALG + 2.5 G & 100 & - & 2.5 \\
4. & ALG: S + 1.5 G & 80 & 20 & 1.5 \\
5. & ALG: S + 2.0 G & 80 & 20 & 2.0 \\
6. & ALG: $+2.5 \mathrm{G}$ & 80 & 20 & 2.5 \\
\hline
\end{tabular}

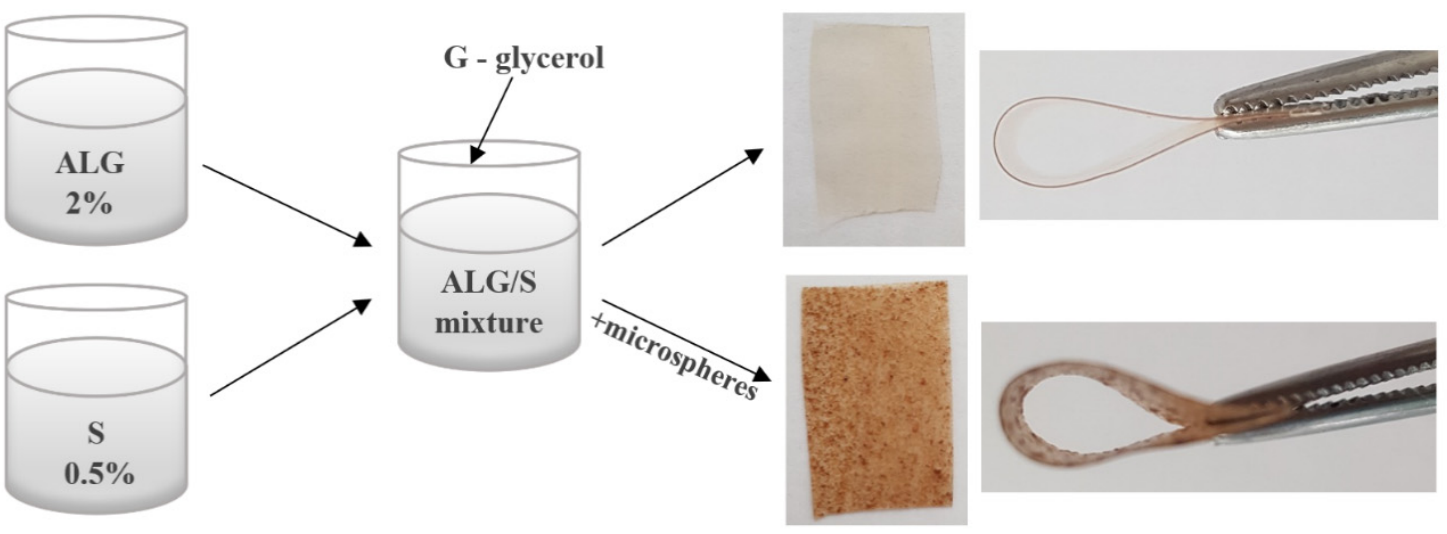

Figure 2. The scheme of production of sodium alginate/starch films with and without microspheres.

\subsection{The Samples Characterization}

\subsubsection{Mechanical Properties}

The mechanical tests were carried out using a mechanical testing machine (Z.05, Zwick/Roell, $\mathrm{Ulm}$, Germany) fitted with a $1 \mathrm{kN}$ load cell. The samples were inserted into the machine clamps and stretched until break with the velocity of $5 \mathrm{~mm} / \mathrm{min}$. The Young's modulus was calculated from the slope of the stress-strain curve in the linear region (strain from $0.25 \%$ to $0.45 \%$ ) using the testXpert program. The tests were carried out in accordance with ASTM D638 standard (ASTM-American Society for Testing and Materials). Five measurements for each type of films were made and average values were calculated.

\subsubsection{Moisture Content}

The samples $(2 \times 2 \mathrm{~cm})$ were weighed and dried $\left(110^{\circ} \mathrm{C}, 24 \mathrm{~h}\right)$ to a constant weight. All samples were weighed again, and the weight loss of each sample was determined. The moisture content was 
calculated as the percentage of water removed from system. The measurements were performed in triplicate [29].

\subsubsection{Contact Angles and Surface Free Energy}

T he contact angles of two liquids, diiodomethane (D) and glycerol (G), on the polymeric films were measured at constant temperature $\left(22^{\circ} \mathrm{C}\right)$ using goniometer equipped with a system of drop shape analysis (DSA 10 produced by Krüss, Hamburg, Germany). The surface free energy was calculated by Owens-Wendt method. The values as average of 10 measurements of each sample were calculated. Due to the significant surface roughness of the films containing microspheres, only films without the addition of microspheres were subjected to contact angle measurements.

\subsubsection{Loading Capacity of Matrices with Microspheres}

The films with extract-loaded gelatin microspheres $(1 \times 1 \mathrm{~cm})$ were weighed and put into $2 \mathrm{~mL}$ of $1 \mathrm{M} \mathrm{NaOH}$ for $1 \mathrm{~h}$. After that, the samples were centrifuged $(10,000 \mathrm{rpm}, 5 \mathrm{~min})$ and the supernatant solution was collected. The polyphenolic compounds were examined by using the Folin-Ciocalteu method [28]. Therefore, $20 \mu \mathrm{L}$ of sample with extract was mixed with $100 \mu \mathrm{L}$ of Folin-Ciocalteu's reagent and $1.58 \mu \mathrm{L}$ of $\mathrm{H}_{2} \mathrm{O}$. After $4 \mathrm{~min}, 300 \mu \mathrm{L}$ of saturated $\mathrm{Na}_{2} \mathrm{CO}_{3}$ solution was added to the mixture. The samples were kept in the incubator at $40{ }^{\circ} \mathrm{C}$ for $30 \mathrm{~min}$. Then, the absorbance was measured at $725 \mathrm{~nm}$ using a UV-VIS spectrophotometer (UV-1800, Shimadzu, Kyoto, Japan). The results are the average of three samples. The presented data of polyphenol content in the matrices with microspheres were calculated based on gallic acid solution using standard curve equation in the concentration range $0-0.50 \mathrm{mg} / \mathrm{mL}$. The coefficient of linear correlation was $\mathrm{R}=0.9992$.

\subsubsection{In Vitro Release Study}

The obtained polymer films with extract-loaded microspheres were placed in a polystyrene 12-well plate and $2 \mathrm{~mL}$ acetate buffer ( $\mathrm{pH}$ 5.4) was added. Then, samples were incubated at $37^{\circ} \mathrm{C}$ and the solution was collected after 30, 60, 90, 120, 180, and $300 \mathrm{~min}$ of incubation. The content of polyphenolic compounds was determined by the Folin-Ciocalteu test [28]. The procedure of preparing samples for in vitro release study was the same as for the loading capacity described above. The absorbance was measured at $725 \mathrm{~nm}$ using a UV-VIS spectrophotometer. The obtained results are the average of measurements taken for three samples of a given type. The experimental calibration curve for the standard solution (gallic acid), prepared previously for loading capacity was used. The release from matrices containing microspheres with the extract was calculated on the basis of the results of loading capacity.

\subsubsection{Skin Examination}

The skin color and TEWL after application of the sodium alginate and sodium alginate/starch films were examined using the colorimeter (Skin-Colorimeter CL 400, Courage + Khazaka, Köln, Germany) and tewameter (Tewameter TM 300, Courage + Khazaka, Köln, Germany) with the use of MPA-software. The films were immersed in water, applied on the skin, and removed after $10 \mathrm{~min}$. The influence of the obtained films on the biophysical skin parameters was determined before application, immediately after application and 15, 30, 60, and $120 \mathrm{~min}$ after application of the samples. The skin parameters measurements were conducted on the volar forearm skin with participation of five probants with normal skin (women, aged 23-25) after informed oral and written consent, in the laboratory in controlled temperature $\left(20-22^{\circ} \mathrm{C}\right)$ and humidity (relative humidity $\left.40-60 \%\right)$. 


\section{Results and Discussion}

\subsection{Mechanical Properties}

The results of the Young's modulus measurements are presented in Table 2. The film thickness was measured before testing. The thickness of the prepared polymer films was from 96 to $120 \mu \mathrm{m}$ (data not shown). The alginate-based films with starch were slightly thicker than films without it. It is known that the mechanical properties of biopolymer films can be improved by the addition of plasticizers, which can bestow flexibility of films due to increase of the free volume or molecular mobility of polymers and decrease the intermolecular forces [30]. The glycerol is the plasticizersamples containing a greater addition of glycerol were more flexible and consequently, their values of Young's modulus were lower, so they broke up later. The values of Young's modulus with the increase in the amount of glycerin decreased from 79.3 to $10.0 \mathrm{MPa}$ and 67.6 to $14.8 \mathrm{MPa}$ for alginate and alginate/starch films, respectively. Gao et al. produced films from sodium alginate and they also observed that the lower values of Young's modulus were due to greater content of glycerol in the materials [31].

Table 2. The values of Young's modulus $(E)$ of sodium alginate-based films modified by the addition of microspheres and unmodified: ALG + 1.5 G, ALG + 2.0 G, ALG + 2.5 G-samples based on sodium alginate containing $1.5 \%, 2 \%$, and $2.5 \%$ of glycerin, respectively. ALG: $\mathrm{S}+1.5 \mathrm{G}, \mathrm{ALG}: \mathrm{S}+2.0 \mathrm{G}$, ALG: $\mathrm{S}+2.5 \mathrm{G}$ samples based on sodium alginate and starch containing $1.5 \%, 2 \%$, and $2.5 \%$ of glycerin, respectively.

\begin{tabular}{ccc}
\hline & \multicolumn{2}{c}{$E \mathbf{~ ( M P a )}$} \\
\hline & Without Microspheres & With Microspheres \\
\hline ALG + 1.5 G & $79.3 \pm 2.1$ & $50.6 \pm 0.9$ \\
ALG + 2.0 G & $18.1 \pm 1.1$ & $10.4 \pm 1.8$ \\
ALG + 2.5 G & $10.0 \pm 1.0$ & $2.7 \pm 0.2$ \\
ALG: +1.5 G & $67.6 \pm 3.6$ & $31.7 \pm 2.1$ \\
ALG: $+2.0 \mathrm{G}$ & $25.6 \pm 1.9$ & $12.1 \pm 0.7$ \\
ALG: $\mathrm{S}+2.5 \mathrm{G}$ & $14.8 \pm 0.7$ & $4.4 \pm 0.3$ \\
\hline
\end{tabular}

The differences between the stiffness of samples without microspheres and films containing microspheres were also noticed. The incorporation of gelatin microspheres into sodium alginate and sodium alginate/starch films led to decrease in values of Young's modulus. Thus, the samples without the microspheres were stiffer than those with the addition of microspheres.

If we compare the composition of the prepared polymer films, we can see that the materials based on sodium alginate and starch were more flexible than those with sodium alginate. These results suggest that the addition of the starch to the composites greatly affected mechanical properties.

\subsection{Moisture Content}

The results of moisture content after drying the samples at $110{ }^{\circ} \mathrm{C}$ during $24 \mathrm{~h}$ are shown in the Table 3. The moisture content strongly depends on the content of the glycerol in the polymeric films. The moisture content of sodium alginate-based films increased with increasing glycerol concentration. This is due to the presence of the glycerol, which acts as a water holding agent [32]. These results show that the films with greater amount of plasticizer had less compact structure and allowed water to get inside. Several investigations reported that the moisture content of polymer films increased by adding more plasticizer to them [33-35]. 
Table 3. The moisture content results of the prepared films based on sodium alginate, starch and glycerol: ALG + 1.5 G, ALG + 2.0 G, ALG + 2.5 G—samples based on sodium alginate containing 1.5\%, $2 \%$, and $2.5 \%$ of glycerin, respectively. ALG: $\mathrm{S}+1.5 \mathrm{G}, \mathrm{ALG}: \mathrm{S}+2.0 \mathrm{G}, \mathrm{ALG}: \mathrm{S}+2.5 \mathrm{G}$ samples based on sodium alginate and starch containing $1.5 \%, 2 \%$, and $2.5 \%$ of glycerin, respectively.

\begin{tabular}{ccc}
\hline & \multicolumn{2}{c}{ Moisture Content (\%) } \\
\hline & Without Microspheres & With Microspheres \\
\hline ALG + 1.5 G & $39.6 \pm 2.7$ & $34.9 \pm 1.2$ \\
ALG + 2.0 G & $50.6 \pm 3.1$ & $42.8 \pm 1.9$ \\
ALG + 2.5 G & $64.8 \pm 4.2$ & $52.6 \pm 0.7$ \\
ALG: S + 1.5 G & $34.4 \pm 3.1$ & $32.5 \pm 1.2$ \\
ALG: + 2.0 G & $44.4 \pm 1.1$ & $43.2 \pm 2.7$ \\
ALG: $S+2.5$ G & $52.4 \pm 3.7$ & $46.9 \pm 2.2$ \\
\hline
\end{tabular}

The addition of starch to the films reduced the moisture content of both matrices, with and without microparticles. Another important observation is that the polymer films containing microspheres showed less moisture content than matrices without them. The highest value of humidity was observed for the film composed of sodium alginate with the addition of $2.5 \%$ glycerol $(\approx 65 \%)$. In turn, the sample containing starch and sodium alginate with $1.5 \%$ of glycerol and microspheres had the lowest water uptake capacity $(\approx 32 \%)$.

\subsection{Contact Angles and Surface Free Energy}

The results of the contact angles, the surface free energy, and its dispersive and polar components calculated using Owens-Wendt method for sodium alginate-based films are shown in Table 4 . The contact angle is a measure of non-covalent forces between first monolayer of material and liquid [36]. The contact angle values for diiodomethane (D) and glycerol (G) of the prepared polymer films were measured. After adding starch to the sodium alginate-based films, we observed decrease in contact angle values for diiodomethane and increase for glycerol compared to the films produced from sodium alginate. In the case of the matrices containing $1.5 \%, 2.0 \%$, and $2.5 \%$ of glycerol, the systematic increase in the contact angle for both glycerol and diiodomethane was noticed.

Table 4. The contact angles $\left(^{\circ}\right)$ of diiodomethane (D) and glycerol $(\mathrm{G})$, the surface free energy $(\gamma)$, and dispersive $\left(\gamma^{\mathrm{d}}\right)$ and polar components $\left(\gamma^{\mathrm{p}}\right)$ values for sodium alginate-based films (calculated by Owens-Wendt method): ALG + 1.5 G, ALG + 2.0 G, ALG + 2.5 G-samples based on sodium alginate containing $1.5 \%, 2 \%$, and $2.5 \%$ of glycerin, respectively. ALG: $\mathrm{S}+1.5 \mathrm{G}, \mathrm{ALG}: \mathrm{S}+2.0 \mathrm{G}, \mathrm{ALG}: \mathrm{S}+2.5 \mathrm{G}$ samples based on sodium alginate and starch containing $1.5 \%, 2 \%$, and $2.5 \%$ of glycerin, respectively.

\begin{tabular}{|c|c|c|c|c|c|}
\hline & \multicolumn{2}{|c|}{ Contact Angle $\left({ }^{\circ}\right)$} & \multirow{2}{*}{$\begin{array}{c}\text { Surface Free } \\
\text { Energy }(\gamma) \\
(\mathrm{mN} / \mathrm{m})\end{array}$} & \multicolumn{2}{|c|}{$\begin{array}{l}\text { Dispersive }\left(\gamma^{\mathrm{d}}\right) \text { and Polar } \\
\left(\gamma^{\mathrm{p}}\right) \text { Components }(\mathrm{mN} / \mathrm{m})\end{array}$} \\
\hline & D & G & & $\gamma^{\mathrm{d}}$ & $\gamma^{\mathrm{p}}$ \\
\hline $\mathrm{ALG}+1.5 \mathrm{G}$ & 82.4 & 46.6 & 47.0 & 6.91 & 40.09 \\
\hline $\mathrm{ALG}+2.0 \mathrm{G}$ & 79.0 & 54.0 & 40.1 & 9.12 & 31.01 \\
\hline $\mathrm{ALG}+2.5 \mathrm{G}$ & 85.1 & 54.2 & 41.2 & 6.57 & 34.58 \\
\hline ALG: $\mathrm{S}+1.5 \mathrm{G}$ & 63.1 & 57.5 & 37.8 & 17.89 & 19.87 \\
\hline ALG: $\mathrm{S}+2.0 \mathrm{G}$ & 61.6 & 61.2 & 35.9 & 19.42 & 16.49 \\
\hline ALG: $\mathrm{S}+2.5 \mathrm{G}$ & 68.9 & 62.1 & 34.2 & 15.49 & 18.75 \\
\hline
\end{tabular}

The values of the contact angle were used to calculate the surface free energy and its polar and dispersive components. Based on the obtained results (Table 4), one can see that the surface free energy of sodium alginate films decreased slightly by adding the starch to the films. The dispersive component increased significantly and simultaneously the polar component of the surface free energy decreased with the starch addition in the films. We can also conclude that the films prepared from sodium alginate 
were more hydrophilic than the matrices based on sodium alginate and starch as the polar component values were higher for the former. It indicates an intermolecular interaction between functional groups in sodium alginate and groups in starch, which led to the existence of a lower number of polar groups on the blend surfaces. However, no significant differences were observed between the dispersive and polar components values of the samples containing different amounts of glycerol.

\subsection{Loading Capacity and In Vitro Release}

The extract-loaded gelatin microspheres were incorporated into the polymer films. Next, the loading capacity of active substance was studied using the Folin-Ciocalteu test by examining the content of polyphenolic compounds in the collected samples.

Based on the obtained results (Figure 3), we noted that the Calendula officinalis flower extract was successfully entrapped in the polymer films containing the gelatin microspheres. An important observation is that the alginate films had the greater loading capacity than the matrices made from alginate and starch. What is more, the higher content of glycerin in the films was associated with a reduced loading of the extract. The amount of polyphenols ranges from 3 to $7 \mathrm{mg} / \mathrm{g}$ depending on the composition of the obtained polymer films. In our previous study [25], we determined the loading capacity of the extract-loaded gelatin microspheres. The results revealed that the pot marigold extract was entrapped in the gelatin microspheres and it was $33 \mathrm{mg} / \mathrm{g}$ based on gallic acid. After immersion, the Calendula officinalis flower extract was absorbed on the surface and inside the pores of the freeze-dried gelatin microspheres. This can be explained by the features of the gelatin, which has a high swelling ability.

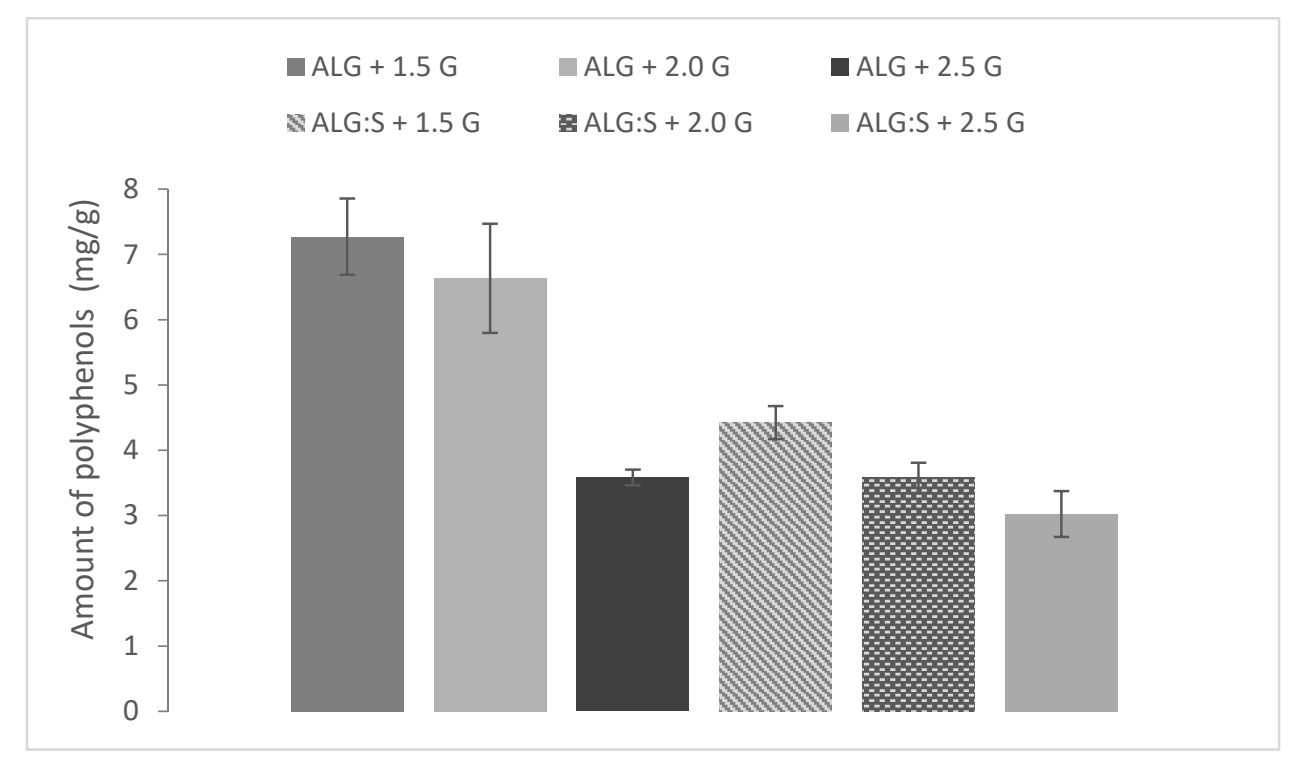

Figure 3. Loading capacity of sodium alginate-based films with loaded gelatin microspheres: ALG + 1.5 G, ALG + 2.0 G, ALG + 2.5 G-samples based on sodium alginate containing $1.5 \%$, $2 \%$, and $2.5 \%$ of glycerin, respectively. ALG: S + 1.5 G, ALG: S+2.0 G, ALG: S + 2.5 G samples based on sodium alginate and starch containing $1.5 \%, 2 \%$, and $2.5 \%$ of glycerin, respectively.

The in vitro release profile of pot marigold flower extract from the obtained materials was studied for $5 \mathrm{~h}$ (Figure 4). We can observe the sustained release of the plant extract. A biphasic pattern of active substance release was noticed for the prepared samples. The prolonged period was followed by a burst effect, during which release of extract significantly accelerated. The first step of releasing the active substance may be associated with the extract diffusion, which was entrapped in the polymer films. In the next phase, the fast release rate was probably caused by swelling of the gelatin microspheres and diffusion of the extract to the release medium [37]. Within the first $180 \mathrm{~min}, \mathrm{max} 15 \%$ of the loaded 
extract was released. During this time, the active substance was released slowly from microspheres loaded into alginate matrix with $2.5 \%$ glycerol content. In the beginning of the test, the release followed slower from matrices made of alginate than films prepared from alginate and starch. After $300 \mathrm{~min}$, the extract was completely released from all samples. This time is sufficient in the case of the application of the prepared materials on the skin.

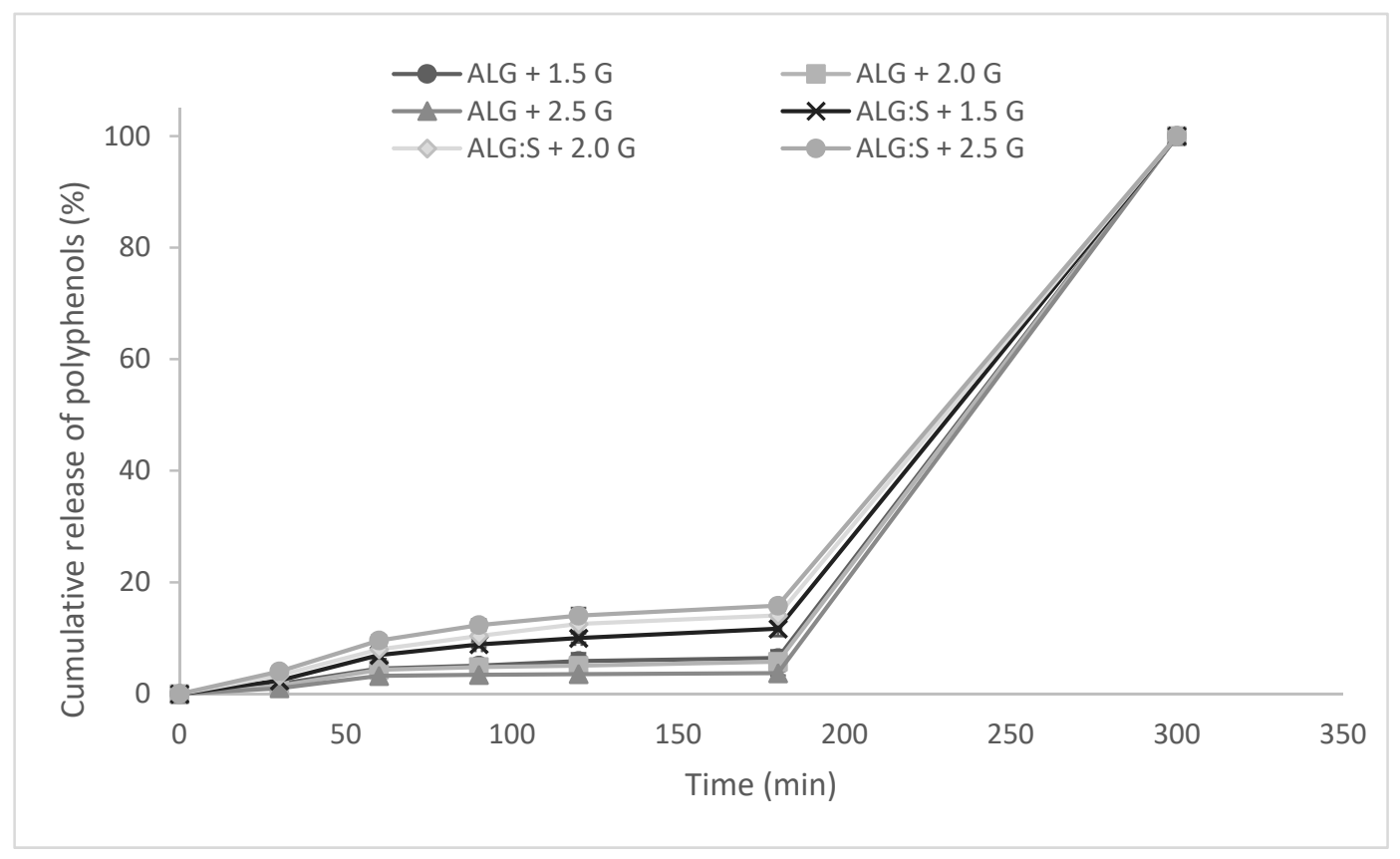

Figure 4. In vitro release assays of sodium alginate-based films modified by the addition of microspheres with encapsulated Calendula officinalis flower extract. ALG + 1.5 G, ALG + 2.0 G, ALG + 2.5 G-samples based on sodium alginate containing $1.5 \%, 2 \%$, and $2.5 \%$ of glycerin, respectively. ALG: S + 1.5 G, ALG: $\mathrm{S}+2.0 \mathrm{G}, \mathrm{ALG}: \mathrm{S}+2.5 \mathrm{G}$ samples based on sodium alginate and starch containing $1.5 \%, 2 \%$, and $2.5 \%$ of glycerin, respectively.

\subsection{Skin Examination}

The results of the analysis of skin parameters after application of polymer films with and without microspheres are shown in Figures 5 and 6. The permeability barrier function was assessed as transepidermal water loss (TEWL). It was observed that the application of materials improved skin's barrier quality manifesting itself in the decrease in TEWL (Figure 5). The use of sodium alginate/starch films caused higher decrease in TEWL in comparison with those composed of sodium alginate. The application of films without microspheres in the structure led to reduction in TEWL immediately and 15 min after materials application, after that time TEWL values remained at stable, lower than the initial level until the end of the study. However, we noticed slight increase in TEWL value immediately after the application of sodium alginate films with the addition of $1.5 \%$ and $2.0 \%$ glycerol, nevertheless, the TEWL values decreased 15 min after application of these matrices. Based on obtained results, we can also conclude that the addition of microspheres into polymer films led to significant decrease in TEWL, which indicates improvement in stratum corneum barrier function. The obtained materials contain glycerol, which acts not only as a moisturizing agent, but also as a promoter of penetration of active substances through the stratum corneum $[38,39]$. Therefore, it was expected that TEWL would decrease due to the presence of glycerol in the materials, which changed the structure of the stratum corneum to allow the Calendula officinalis flower extract to penetrate into deeper skin layers. ALG: S + 2.5 G sample had the greatest effectiveness in improving the integrity of the epidermal barrier. A total of 120 min after applying this sample on the skin, the TEWL was $0.7 \mathrm{~g} / \mathrm{h} / \mathrm{m}^{2}$. 


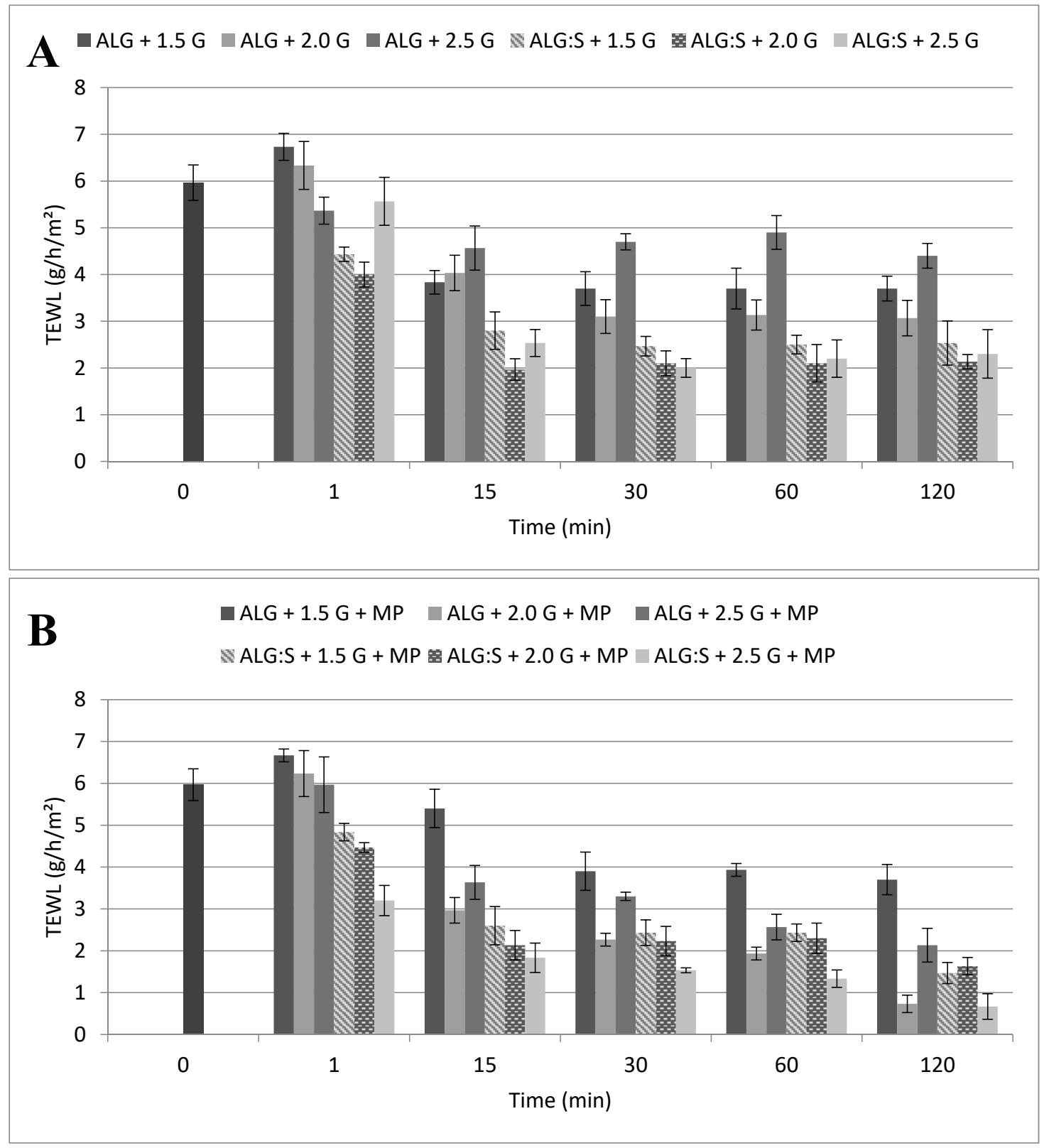

Figure 5. Skin barrier quality (transepidermal water loss-TEWL) after application of sodium alginate-based films (A) without and (B) with microspheres containing encapsulated Calendula officinalis flower extract: ALG + 1.5 G, ALG + 2.0 G, ALG + 2.5 G-samples based on sodium alginate containing $1.5 \%, 2 \%$, and $2.5 \%$ of glycerin, respectively. ALG: S + 1.5 G, ALG: S + 2.0 G, ALG: S + 2.5 G samples based on sodium alginate and starch containing $1.5 \%, 2 \%$, and $2.5 \%$ of glycerin, respectively.

The measured skin color is expressed as an XYZ-value and was calculated into $L^{*} a^{*} b$ related value. $\mathrm{L}^{*}$ gives information about the black-white axis and skin brightness, while $\mathrm{a}^{*}$ and $\mathrm{b}^{*}$ are the coordinates in the color space- $\mathrm{a}^{*}$ locates the values on the red-green axis, whereas $\mathrm{b}^{*}$ shows the color position on the blue-yellow axis. Therefore, the $\mathrm{a}^{*}$ values were considered in this research due to their correlation to skin redness, erythema, and microcirculation. Skin color results suggest that the application of sodium alginate-based films did not damage, irritate skin, or cause erythema (skin redness) (Figure 6). However, slight differences in skin color was initially noticed on skin areas after application of microspheres-loaded films. Graziola et al. produced gelatin-based microspheres and demonstrated that aqueous dispersions of microspheres had good skin compatibility—they did 
not observe an increase in TEWL, which would indicate neither damage to the skin nor break of the barrier function [40].

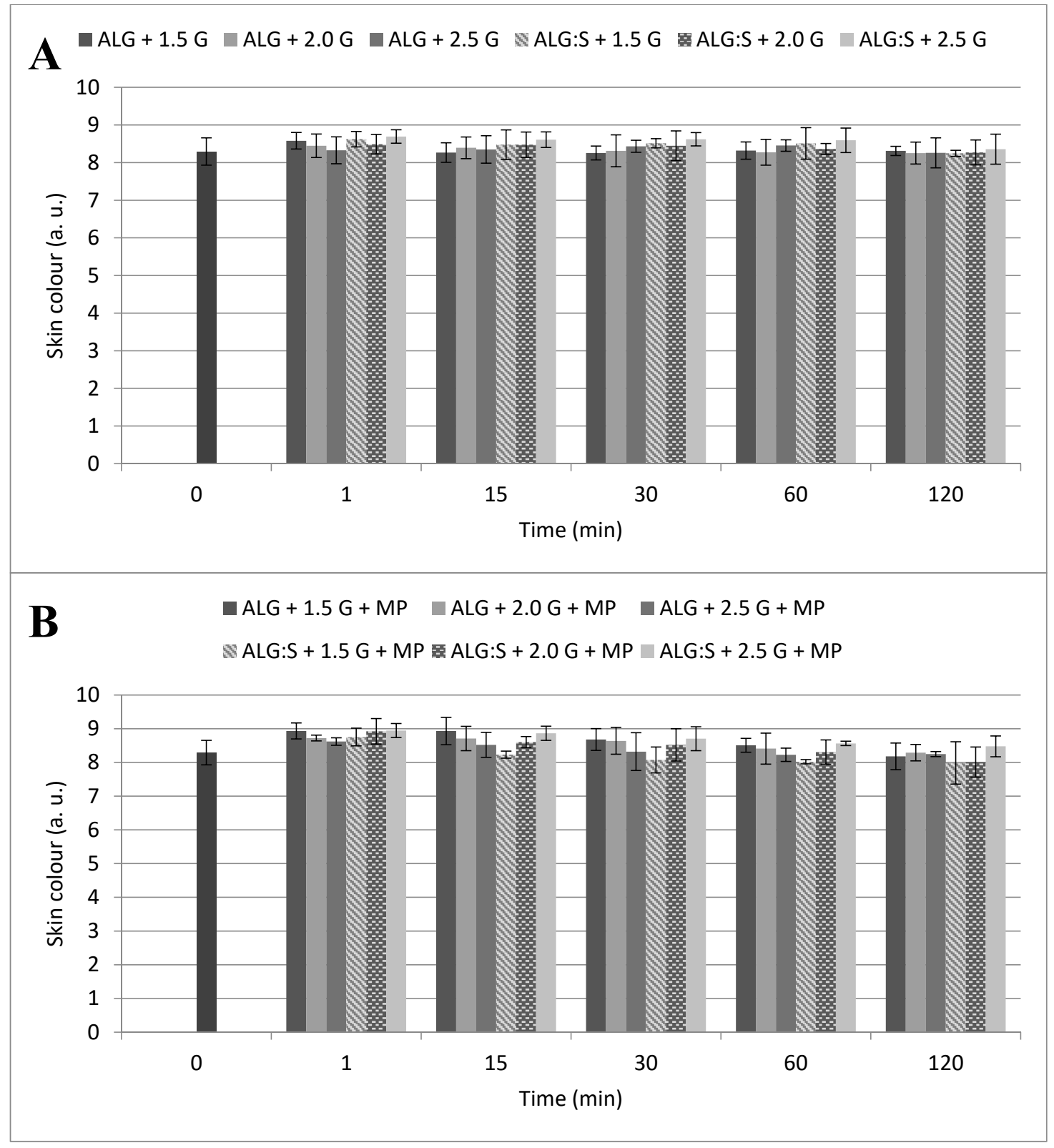

Figure 6. Skin color after application of sodium alginate and sodium alginate/starch films (A) without and (B) with microspheres in the structure: ALG + 1.5 G, ALG + 2.0 G, ALG + 2.5 G-samples based on sodium alginate containing $1.5 \%, 2 \%$, and $2.5 \%$ of glycerin, respectively. ALG: $\mathrm{S}+1.5 \mathrm{G}$, ALG: S + 2.0 G, ALG: S + 2.5 G samples based on sodium alginate and starch containing 1.5\%, $2 \%$, and $2.5 \%$ of glycerin, respectively.

The beneficial effect of materials based on sodium alginate or sodium alginate and starch on biophysical skin parameters is caused by the whole structure of these materials. Moist polymer matrices loosens the skin structure and increases its permeability owing to occlusion and hydrophilicity of polymers. Materials are also modified by the addition of glycerin which act as a penetration promoter-it supports transepidermal penetration, and thus reduces the skin barrier functions. It contributes to the more effective penetration to the deeper skin layers of Calendula officinalis flower extract released from the microparticles. Calendula officinalis flower extract has been well known for 
a long time for its strong healing effects on the skin. Therefore, treatment efficiency of many skin conditions is increased.

\section{Conclusions}

The results of the current work demonstrated that the modification of polymer films by the addition of microspheres with encapsulated Calendula officinalis flower extract has contributed to obtaining materials that have a positive effect on the quality of the skin barrier. The active substance was released slowly from obtained films. The addition of microspheres to the films led to changes in their physicochemical properties, e.g., the values of Young's modulus and the moisture content were decreased. However, deterioration of the functional features of the films was not observed and swollen films could be easily applied on the skin. The most important, the application of obtained materials allows increased protection against transepidermal water loss. Sodium alginate/starch-based film containing $2.5 \%$ of glycerol and microspheres was the most effective in limiting TEWL.

Author Contributions: Conceptualization, J.K.; investigation, N.S., W.P.-W.; resources, J.K., B.T.; data curation J.K., N.S., W.P.-W.; writing—original draft preparation, J.K., N.S., W.P.-W.; writing—review and editing, J.K., B.T.; supervision, J.K.; project administration, J.K.; funding acquisition, J.K. All authors have read and agreed to the published version of the manuscript.

Funding: Financial support from National Science Centre (NCN, Poland) Grant no. UMO-2016/21/D/ST8/01705 is gratefully acknowledged.

Acknowledgments: The authors gratefully acknowledge Jessica Ferreira De Sousa for her support with laboratory work.

Conflicts of Interest: The authors declare no conflict of interest. The funders had no role in the design of the study; in the collection, analyses, or interpretation of data; in the writing of the manuscript, or in the decision to publish the results.

\section{References}

1. Milani, M.; Sparavigna, A. The 24-hour skin hydration and barrier function effects of a hyaluronic $1 \%$, glycerin 5\%, and Centella asiatica stem cells extract moisturizing fluid: An intra-subject, randomized, assessor-blinded study. Clin. Cosmet. Investig. Dermatol. 2017, 10, 311-315. [CrossRef]

2. van Jansen Rensburg, S.; Franken, A.; Du Plessis, J.L. Measurement of transepidermal water loss, stratum corneum hydration and skin surface $\mathrm{pH}$ in occupational settings: A review. Skin Res. Technol. 2019, 25, 595-605. [CrossRef]

3. Engebretsen, K.A.; Johansen, J.D.; Kezic, S.; Linneberg, A.; Thyssen, J.P. The effect of environmental humidity and temperature on skin barrier function and dermatitis. J. Eur. Acad. Dermatol. Venereol. 2016, 30, $223-249$. [CrossRef]

4. Wallen-Russell, C. Is There a Relationship between Transepidermal Water Loss and Microbial Biodiversity on the Skin? Cosmetics 2019, 6, 18. [CrossRef]

5. Biliaderis, C.G. Structural transitions and related physical properties of starch. In Starch, 3rd ed.; James BeMiller, J., Whistler, R., Eds.; Academic Press: Burlington, MA, USA, 2009; pp. 293-372.

6. Mackie, W.; Noy, R.; Sellen, D.B. Solution properties of sodium alginate. Biopolym. Orig. Res. Biomol. 1980, 19, 1839-1860. [CrossRef]

7. Tønnesen, H.H.; Karlsen, J. Alginate in drug delivery systems. Drug Dev. Ind. Pharm. 2002, 28, 621-630. [CrossRef]

8. Pereira, L.; Sousa, A.; Coelho, H.; Amado, A.M.; Ribeiro-Claro, P.J. Use of FTIR, FT-Raman and 13C-NMR spectroscopy for identification of some seaweed phycocolloids. Biomol. Eng. 2003, 20, 223-228. [CrossRef]

9. Tester, R.F.; Karkalas, J.; Qi, X. Starch—composition, fine structure and architecture. J. Cereal Sci. 2004, 39, 151-165. [CrossRef]

10. Buléon, A.; Colonna, P.; Planchot, V.; Ball, S. Starch granules: Structure and biosynthesis. Int. J. Biol. Macromol. 1998, 23, 85-112. [CrossRef]

11. Talja, R.A.; Helén, H.; Roos, Y.H.; Jouppila, K. Effect of various polyols and polyol contents on physical and mechanical properties of potato starch-based films. Carbohydr. Polym. 2007, 67, 288-295. [CrossRef] 
12. Haug, I.J.; Draget, K.I.; Smidsrød, O. Physical and rheological properties of fish gelatin compared to mammalian gelatin. Food Hydrocoll. 2004, 18, 203-213. [CrossRef]

13. Gómez-Estaca, J.; Montero, P.; Fernández-Martín, F.; Gómez-Guillén, M.C. Physico-chemical and film-forming properties of bovine-hide and tuna-skin gelatin: A comparative study. J. Food Eng. 2009, 90, 480-486. [CrossRef]

14. Marturano, V.; Kozlowska, J.; Bajek, A.; Giamberini, M.; Ambrogi, V.; Cerruti, P.; Garcia-Valls, R.; Montornes, J.M.; Tylkowski, B. Photo-triggered capsules based on lanthanide-doped upconverting nanoparticles for medical applications. Coord. Chem. Rev. 2019, 398, 213013. [CrossRef]

15. El Asbahani, A.; Miladi, K.; Badri, W.; Sala, M.; Addi, E.A.; Casabianca, H.; El Mousadik, A.; Hartmann, D.; Jilale, A.; Renaud, F.N.R.; et al. Essential oils: From extraction to encapsulation. Int. J. Pharm. 2015, 483, 220-243. [CrossRef]

16. Delmote, J.; Teruel-Biosca, L.; Ribelles, J.L.G.; Ferrer, G.G. Emulsion based microencapsulation of proteins in poly (L-lactic acid) films and membranes for the controlled release of drugs. Polym. Degrad. Stabil. 2017, 146, 24-33. [CrossRef]

17. Re, T.A.; Mooney, D.; Antignac, E.; Dufour, E.; Bark, I.; Srinivasan, V.; Nohynek, G. Application of the threshold of toxicological concern approach for the safety evaluation of calendula flower (Calendula officinalis) petals and extracts used in cosmetic and personal care products. Food Chem. Toxicol. 2009, 47, 1246-1254. [CrossRef]

18. Preethi, K.C.; Kuttan, G.; Kuttan, R. Antioxidant Potential of an Extract of Calendula officinalis. Flowers in Vitro and in Vivo. Pharm. Biol. 2006, 44, 691-697. [CrossRef]

19. Fonseca, Y.M.; Catini, C.D.; Vicentini, F.T.; Nomizo, A.; Gerlach, R.F.; Fonseca, M.J.V. Protective effect of Calendula officinalis extract against UVB-induced oxidative stress in skin: Evaluation of reduced glutathione levels and matrix metalloproteinase secretion. J. Ethnopharmacol. 2010, 127, 596-601. [CrossRef]

20. Ukiya, M.; Akihisa, T.; Yasukawa, K.; Tokuda, H.; Suzuki, T.; Kimura, Y. Anti-inflammatory, anti-tumor-promoting, and cytotoxic activities of constituents of marigold (Calendula officinalis) flowers. J. Nat. Prod. 2006, 69, 1692-1696. [CrossRef]

21. Efstratiou, E.; Hussain, A.I.; Nigam, P.S.; Moore, J.E.; Ayub, M.A.; Rao, J.R. Antimicrobial activity of Calendula officinalis petal extracts against fungi, as well as Gram-negative and Gram-positive clinical pathogens. Complement. Ther. Clin. Prac. 2012, 18, 173-176. [CrossRef]

22. Jiménez-Medina, E.; Garcia-Lora, A.; Paco, L.; Algarra, I.; Collado, A.; Garrido, F. A new extract of the plant calendula officinalis produces a dual in vitroeffect: Cytotoxic anti-tumor activity and lymphocyte activation. BMC Cancer 2006, 6, 119. [CrossRef] [PubMed]

23. Chandran, P.K.; Kuttan, R. Effect of Calendula officinalis flower extract on acute phase proteins, antioxidant defense mechanism and granuloma formation during thermal burns. J. Clin. Biochem. Nutr. 2008, 43, 58-64. [CrossRef] [PubMed]

24. Jan, N.; John, R. Calendula officinalis-an important medicinal plant with potential biological properties. Proc. Indian Natl. Sci. Acad. 2017, 83, 769-787.

25. Kozlowska, J.; Stachowiak, N.; Prus, W. Stability studies of collagen-based microspheres with Calendula officinalis flower extract. Polym. Degrad. Stabil. 2019, 163, 214-219. [CrossRef]

26. Kozlowska, J.; Stachowiak, N.A.; Sionkowska, A. The preparation and characterization of composite materials by incorporating microspheres into a collagen/hydroxyethyl cellulose matrix. Polym. Test. 2018, 69, 350-358. [CrossRef]

27. Kozlowska, J.; Stachowiak, N.; Sionkowska, A. Collagen/gelatin/hydroxyethyl cellulose composites containing microspheres based on collagen and gelatin: Design and evaluation. Polymers 2018, 10, 456. [CrossRef]

28. Singleton, V.L.; Rossi, J.A. Colorimetry of total phenolics with phosphomolybdic-phosphotungstic acid reagents. Am. J. Enol. Vitic. 1965, 16, 144-158.

29. Aguirre-Loredo, R.Y.; Rodríguez-Hernández, A.I.; Morales-Sánchez, E.; Gómez-Aldapa, C.A.; Velazquez, G. Effect of equilibrium moisture content on barrier, mechanical and thermal properties of chitosan films. Food Chem. 2016, 196, 560-566. [CrossRef]

30. Senturk Parreidt, T.; Müller, K.; Schmid, M. Alginate-based edible films and coatings for food packaging applications. Foods 2018, 7, 170. [CrossRef]

31. Gao, C.; Pollet, E.; Avérous, L. Properties of glycerol-plasticized alginate films obtained by thermo-mechanical mixing. Food Hydrocoll. 2017, 63, 414-420. [CrossRef] 
32. Tapia-Blácido, D.R.; do Amaral Sobral, P.J.; Menegalli, F.C. Effect of drying conditions and plasticizer type on some physical and mechanical properties of amaranth flour films. LWT-Food Sci. Technol. 2013, 50, 392-400. [CrossRef]

33. Ghasemlou, M.; Khodaiyan, F.; Oromiehie, A. Physical, mechanical, barrier, and thermal properties of polyol-plasticized biodegradable edible film made from kefiran. Carbohydr. Polym. 2011, 84, 477-483. [CrossRef]

34. Zhang, Y.; Han, J.H. Plasticization of pea starch films with monosaccharides and polyols. J. Food Sci. 2006, 71, E253-E261. [CrossRef]

35. Shaw, N.B.; Monahan, F.J.; O'riordan, E.D.; O'sullivan, M. Physical properties of WPI films plasticized with glycerol, xylitol, or sorbitol. J. Food Sci. 2002, 67, 164-167. [CrossRef]

36. Sionkowska, A.; Płanecka, A.; Kozłowska, J.; Skopińska-Wiśniewska, J. Surface properties of UV-irradiated poly (vinyl alcohol) films containing small amount of collagen. Appl. Surf. Sci. 2009, 255, 4135-4139. [CrossRef]

37. Tran, T.H.; Ramasamy, T.; Poudel, B.K.; Marasini, N.; Moon, B.K.; Cho, H.J.; Choi, H.G.; Yong, C.S.; Kim, J.O. Preparation and characterization of spray-dried gelatin microspheres encapsulating ganciclovir. Macromol. Res. 2014, 22, 124-130. [CrossRef]

38. Batt, M.D.; Fairhurst, E. Hydration of the stratum corneum. Int. J. Cosmet. Sci. 1986, 8, 253-264. [CrossRef]

39. Lodén, M. Effect of moisturizers on epidermal barrier function. Clin. Dermatol. 2012, 30, 286-296. [CrossRef]

40. Graziola, F.; Candido, T.M.; de Oliveira, C.A.; Peres, D.D.A.; Issa, M.G.; Mota, J.; Rosado, C.; Consiglieri, V.O.; Kaneko, T.M.; Velasco, M.V.R.; et al. Gelatin-based microspheres crosslinked with glutaraldehyde and rutin oriented to cosmetics. Braz. J. Pharm. Sci. 2016, 52, 603-612. [CrossRef]

(C) 2020 by the authors. Licensee MDPI, Basel, Switzerland. This article is an open access article distributed under the terms and conditions of the Creative Commons Attribution (CC BY) license (http://creativecommons.org/licenses/by/4.0/). 